\title{
IRIS Based Human Recognition System
}

\author{
Mansi Jhamb \\ USIT, Guru Gobind Singh Indraprastha \\ University, Delhi, India
Vinod Kumar Khera
Guru Tegh Bahadur Institute of
Technology Guru Gobind Singh \\ Indraprastha University, Delhi, India
}

mansi.jhamb@gmail.com

vinodkhera@gmail.com

\begin{abstract}
The paper explores iris recognition for personal identification and verification. In this paper a new iris recognition technique is proposed using (Scale Invariant Feature Transform) SIFT. Imageprocessing algorithms have been validated on noised real iris image database. The proposed innovative technique is computationally effective as well as reliable in terms of recognition rates.
\end{abstract}

Keywords: Iris Recognition, Hough Transform, SIFT, Key-Points

\section{INTRODUCTION}

Today, biometric recognition is a common and reliable way to authenticate the identity of a living person based on physiological or behavioral characteristics. A physiological characteristic is relatively stable physical characteristics, such as fingerprint, iris pattern, facial feature, hand silhouette, etc. This kind of measurement is basically unchanging and unalterable without significant duress. A behavioral characteristic is more a reflection of an individual's psychological makeup as signature, speech pattern, or how one types at a keyboard. The degree of intrapersonal variation in a physical characteristic is smaller than a behavioral characteristic. For examples, a signature is influenced by both controllable actions and less psychological factors, and speech pattern is influenced by current emotional state, whereas fingerprint template is independent. Nevertheless all physiology-based biometrics don't offer satisfactory recognition rates (false acceptance and/or false reject rates, respectively referenced as FAR and FRR). The automated personal identity authentication systems based on iris recognition are reputed to be the most reliable among all biometric methods: we consider that the probability of finding two people with identical iris pattern is almost zero [1]. That's why iris recognition technology is becoming an important biometric solution for people identification in access control as networked access to computer application [2]. Compared to fingerprint, iris is protected from the external environment behind the cornea and the eyelid. No subject to deleterious effects of aging, the small-scale radial features of the iris remain stable and fixed from about one year of age throughout life. This paper is divided into 4 main parts. The Section 1 introduces what is the position of iris technology in personal authentication. In the Section 2, we sum up the state of the art in the domain of iris recognition. The more widely known iris recognition system developed by J.Daugman [4] is taken as reference for comparison. The Section 3 presents in details our approach, and discusses the different issues we chose. At last a conclusion is done in Section 4, which tasks about the next considerations for the improvement of the proposed solution.

\section{LITERATURE SURVEY}

The French ophthalmologist Alphonse Bertillon seems to be the first to propose the use of iris pattern (color) as a basis for personal identification [3]. In 1981, after reading many scientific reports describing the iris great variation, Flom and San Francisco ophthalmologist Aran Safir suggested also using the iris as the basis for a biometric. In 1987, they began collaborating with computer scientist John Daugman of Cambridge University in England to develop iris identification software who published his first promising results in 1992 [4]. Later on a little similar 
works have been investigated, such as R.Wildes' [5], W.Boles' [6] and R.Sanchez- Reillo's [7] systems, which differ both in the iris features representation (iris signature) and pattern matching algorithms. R.Wildes' solution includes (i) a Hough transform for iris localization, (ii) Laplacian pyramid(multi-scale decomposition) to represent distinctive spatial characteristics of the human iris, and (iii) modified normalized correlation for matching process. W.Boles' prototype operates in building (j) a one dimensional representation of the gray level profiles of the iris followed by obtaining the wavelet transform zero-crossings of the resulting representation, and (jj) original dissimilarity functions that enable pertinent information selection for efficient matching computation. To finish J.Daugman's and R.Sanchez-Reillo's systems are implemented exploiting (I) integrodifferential operators to detect iris inner and outer boundaries, (II) Gabor filters to extract unique binary vectors constituting iriscodeTM, and (III) a statistical matcher (logical exclusive OR operator) that analyses basically the average Hamming distance between two codes (bit to bit test agreement). Because of unified reference database of iris images does not exist, a classic performance comparison of the described systems is not trivial. However in terms of recognition rates (FAR, FRR), the commercial success of the patented Daugman's system speak in his favor. Indeed Daugman's mathematical algorithms have been contributing to a commercial solution patented by IriScan Inc. This biometric identification platform processes iris recognition through (i) a specific optical unit that enables noninvasive acquisition of iris images, and (ii) a data processing unit. Although capturing a well-defined image of the iris while not interacting actively with the device seems to be one the major challenge we encountered for iris recognition system design, our research focus on the second block both in charge of (j) the enrolment process, and (jj) the matching which quantifies the similitude between two biometric templates.

\section{PROPOSED APPROACH}

Previous work on iris recognition, derived from the information found in the open literature, led us to suggest a few possible improvements. For justification of these new concepts we implemented in Matlab/C . The algorithm used is as follows:

- Image Acquisition

- Iris Localization.

- Find the darkest point of image (referred as black hole) in the global image analysis.

- Determine a range of darkness (based on 1) designated as the threshold value $(t)$ for identification of black holes.

- Determine the number of black holes and their coordinates according to the predefined threshold. Calculate the centre of mass of these black holes.

- Construct a $L \times L$ region centred at the estimated centroid.

- Repeat step 3 to improve the estimation of actual centroid of pupil.

- $\quad$ Find key points using SIFT.

- Match the key points of the input image with the key points of images in database.

The algorithm is beautifully explained by following algorithmic flow chart ,figure 1

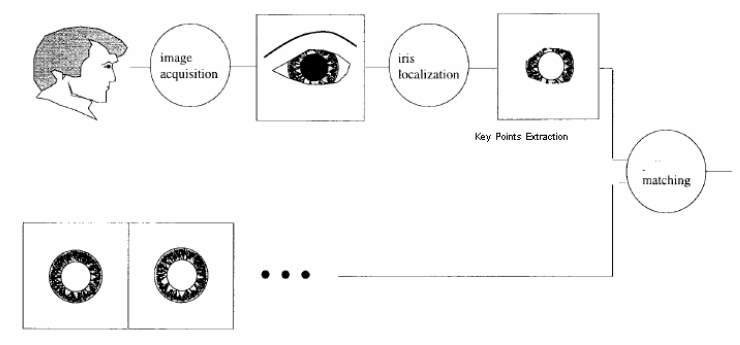

FIGURE 1: Iris Recognition: The Process

\subsection{IMAGE ACQUISITION}

One of the major challenges of automated iris recognition is to capture a high-quality image of the iris while remaining noninvasive to the human operator. Given that the iris is a relatively small (typically about $1 \mathrm{~cm}$ in diameter), dark object and that human operators are very sensitive about 
their eyes, this matter requires careful engineering. Several points are of particular concern. First, it is desirable to acquire images of the iris with sufficient resolution and sharpness to support recognition. Second, it is important to have good contrast in the interior iris pattern without resorting to a level of illumination that annoys the operator, i.e., adequate intensity of source $(\mathrm{W} / \mathrm{cm})$ constrained by operator comfort with brightness $(\mathrm{W} / \mathrm{sr}-\mathrm{cm})$. Third, these images must be well framed (i.e., centered) without unduly constraining the operator (i.e., preferably without requiring the operator to employ an eye piece, chin rest, or other contact positioning that would be invasive). Further, as an integral part of this process, artifacts in the acquired images (e.g., due to specular reflections, optical aberrations, etc.) should be eliminated as much as possible. Schematic diagrams of two image-acquisition rigs that have been developed in response to these challenges. The acquired Image is as shown in figure 2 below:

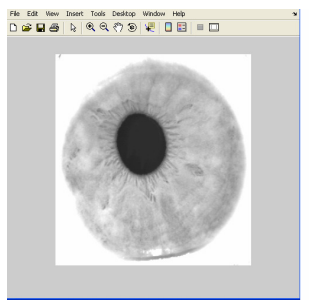

FIGURE 2: Acquired Image

\subsection{IRIS LOCALIZATION}

Without placing undue constraints on the human operator, image acquisition of the iris cannot be expected to yield an image containing only the iris. Rather, image acquisition will capture the iris as part of a larger image that also contains data derived from the immediately surrounding eye region. Therefore, prior to performing iris pattern matching, it is important to localize that portion of the acquired image that corresponds to an iris. In particular, it is necessary to localize that portion of the image derived from inside the limbus (the border between the sclera and the iris) and outside the pupil. Further, if the eyelids are occluding part of the iris, then only that portion of the image below the upper eyelid and above the lower eyelid should be included. Typically, the limbic boundary is imaged with high contrast, owing to the sharp change in eye pigmentation that it marks. The upper and lower portions of this boundary, however, can be occluded by the eyelids. The papillary boundary can be far less well defined. The image contrast between a heavily pigmented iris and its pupil can be quite small. Further, while the pupil typically is darker than the iris, the reverse relationship can hold in cases of cataract: the clouded lens leads to a significant amount of backscattered light. Like the pupillary boundary, eyelid contrast can be quite variable depending on the relative pigmentation in the skin and the iris. The eyelid boundary also can be irregular due to the presence of eyelashes. Taken in tandem, these observations suggest that iris localization must be sensitive to a wide range of edge contrasts, robust to irregular borders, and capable of dealing with variable occlusion. The systems differ mostly in the way that they search their parameter spaces to fit the contour models to the image information. To understand how these searches proceed, let $\mathrm{I}(\mathrm{x}, \mathrm{y})$ represent the image intensity value at location $(\mathrm{x}, \mathrm{y})$ and let circular contours (for the limbic and papillary boundaries) be parameterized by center location (xc,yc) and radius $\mathrm{r}$. The Daugman system fits the circular contours via gradient ascent on the parameters $(x c, y c, r)$ so as to maximize

$$
\left|\frac{\partial}{\partial r} G(r) * \oint_{r, x_{c}, y_{c}} \frac{I(x, y)}{2 \pi r} d s\right|
$$

Where $G(r)=(1 / \sqrt{2 \sigma \Pi}) \sigma^{-(r-r o) 2} / 2 \sigma^{2}$ is a radial Gaussian with center ro and standard deviation $\sigma$ that smooths the image to select the spatial scale of edges under consideration * symbolizes convolution, ds is an element of circular arc, and division by $2 \pi r$ serves to normalize the integral. In order to incorporate directional tuning of the image derivative, the arc of integration ds is restricted to the left and right quadrants (i.e., near vertical edges) when fitting the limbic boundary. This arc is considered over a fuller range when fitting the pupillary boundary; however, 
the lower quadrant of the image is still omitted due to the artifact of the specular reflection of the illuminant in that region (see Section II-A). In implementation, the contour fitting procedure is discretized, with finite differences serving for derivatives and summation used to instantiate integrals and convolutions. More generally, fitting contours to images via this type of optimization formulation is a standard machine vision technique, often referred to as active contour modeling The Wildes et al. system performs its contour fitting in two steps. First, the image intensity information is converted into a binary edge-map. Second, the edge points vote to instantiate particular contour parameter values. The edgemap is recovered via gradient-based edge detection [2], [44]. This operation consists of thresholding the magnitude of the image intensity gradient, i.e.,

$$
\begin{gathered}
\nabla G(x, y) * I(x, y) \text { where } \\
\nabla \equiv(\partial / \partial x, \partial / \partial y) \text { while } \\
G(x, y)=1 / 2 \Pi \sigma^{2} e-{ }^{(x-x 0)^{2}+(r-r o)^{2} / 2 \sigma^{2}}
\end{gathered}
$$

is a two-dimensional Gaussian with center (xo,yo) and $\sigma$ is standard deviation that smooths the image to select the spatial scale of edges under consideration. In order to incorporate directional tuning, the image intensity derivatives are weighted to favor certain ranges of orientation prior to taking the magnitude. For example, prior to contributing to the fit of the limbic boundary contour, the derivatives are weighted to be selective for vertical edges. The voting procedure is realized via Hough transforms [27], [28] on parametric definitions of the iris boundary contours. In particular, for the circular limbic or pupillary boundaries and a set of recovered edge points $(x \mathrm{j}, \mathrm{yj}) \mathrm{j}$ $=1 \ldots . . \mathrm{n}$. Hough transform is defined as

$$
H\left(x_{c}, y_{c}, r\right)=\sum_{j=1}^{n} h\left(x_{j}, y_{j}, x_{c}, y_{c}, r\right)
$$

where

$$
h\left(x_{j}, y_{j}, x_{c}, y_{c}, r\right)= \begin{cases}1, & \text { if } g\left(x_{j}, y_{j}, x_{c}, y_{c}, r\right)=0 \\ 0, & \text { otherwise }\end{cases}
$$

with

$$
g\left(x_{j}, y_{j}, x_{c}, y_{c}, r\right)=\left(x_{j}-x_{c}\right)^{2}+\left(y_{j}-y_{c}\right)^{2}-r^{2} .
$$

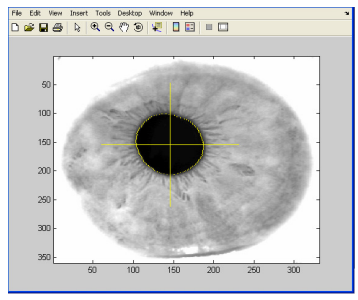

FIGURE 3: Iris and centroid detection

\subsection{IRIS MATCHING}

Image matching is a fundamental aspect of many problems in computer vision, including object or scene recognition, solving for 3D structure from multiple images, stereo correspondence, and motion tracking. This method describes image features that have many properties that make them suitable for matching differing images of an object or scene. The features are invariant to image scaling and rotation, and partially invariant to change in illumination and 3D camera viewpoint. They are well localized in both the spatial and frequency domains, reducing the probability of disruption by occlusion, clutter, or noise. Large numbers of features can be extracted from typical images with efficient algorithms. In addition, the features are highly distinctive, which allows a single feature to be correctly matched with high probability against a large database of features, providing a basis for object and scene recognition. The cost of 
extracting these features is minimized by taking a cascade filtering approach, in which the more expensive operations are applied only at locations that pass an initial test.

Following are the major stages of computation used to generate the set of image features:

- Scale-space extrema detection:

- Key point localization:

- Orientation assignment:

- Key point descriptor:

\subsubsection{Detection Of Scale Space Schema}

As described in the introduction, we will detect keypoints using a cascade filtering approach that uses efficient algorithms to identify candidate locations that are then examined in further detail. The first stage of keypoint detection is to identify locations and scales that can be repeatably assigned under differing views of the same object. Detecting locations that are invariant to scale change of the image can be accomplished by searching for stable features across all possible scales, using a continuous function of scale known as scale space (Witkin,1983).It has been shown by Koenderink (1984) and Lindeberg (1994) that under a variety of reasonable assumptions the only possible scale-space kernel is the Gaussian function. Therefore, the scale space of an image is defined as a function, $L(x, y, \sigma)$, that is produced from the convolution of a variable-scale Gaussian, $G(x, y, \sigma)$, with an input image, $I(x, y)$ :

Where ${ }^{*}$ is the convolution operation in $x$ and $y$,

$$
L(x, y, \sigma)=G(x, y, \sigma){ }^{*} I(x, y) \text {, }
$$

$$
G(x, y, \sigma)=\frac{1}{2 \pi \sigma^{2}} e^{-\left(x^{2}+y^{2}\right) / 2 \sigma^{2}}
$$

and To efficiently detect stable keypoint locations in scale space, we have proposed (Lowe, 1999) using scale-space extrema in the difference-of-Gaussian function convolved with the image, $D(x$, $y, \sigma)$ which can be computed from the difference of two nearby scales separated by a constant multiplicative factor $\mathrm{k}$ :

$$
D(x, y, \sigma)=\left(G(x, y, k \sigma)-G(x, y, \sigma){ }^{*} I(x, y)=L(x, y, k \sigma)-L(x, y, \sigma)\right.
$$

There are a number of reasons for choosing this function. First, it is a particularly efficient function to compute, as the smoothed images, L, need to be computed in any case for scale space feature description, and $\mathrm{D}$ can therefore be computed by simple image subtraction In addition, the difference-of-Gaussian function provides a close approximation to the scale-normalized Laplacian of Gaussian, $\sigma 2 \nabla^{2} G$, as studied by Lindeberg (1994). Lindeberg showed that the normalization of the Laplacian with the factor $\sigma^{2}$ is required for true scale invariance. The relationship between $D$ and $\sigma^{2} \nabla^{2} G$ can be understood from the heat diffusion equation (parameterized in terms of $\sigma$ rather than the more usual $\left.\mathrm{t}=\sigma^{2}\right): \partial G / \partial \sigma=\sigma \nabla^{2} G$

From this, we see that $\nabla^{2} G$ can be computed from the finite difference approximation to $d G / d \sigma$, using the difference of nearby scales at $\mathrm{k} \sigma$ and $\sigma$ :

$$
\sigma \nabla^{2} G=\partial G / \partial \sigma \approx G(x, y, k \sigma)-G(x, y, \sigma) / K \sigma-\sigma
$$

And therefore

$$
\sigma \nabla^{2} G=\partial G / \partial \sigma \approx G(x, y, k \sigma) \approx(k-1) \sigma^{2} \nabla^{2} G \text {. }
$$

The factor $(k-1)$ in the equation is aconstant over all scales and therefore does not influence extrema location. An important aspect of this approach is that it generates large numbers of features that densely cover the image over the full range of scales and locations. For iris matching and recognition, SIFT features are first extracted from a set of reference images and stored in a database.

\subsubsection{Accurate Key-point Localization}

Once a keypoint candidate has been found by comparing a pixel to its neighbors, the next step is to perform a detailed fit to the nearby data for location, scale, and ratio of principal curvatures. This information allows points to be rejected that have low contrast (and are therefore sensitive to noise) or are poorly localized along an edge. The initial implementation of this approach (Lowe, 
1999) simply located keypoints at the location and scale of the central sample point. However, recently Brown has developed a method (Brown and Lowe, 2002) for fitting a 3D quadratic function to the local sample points to determine the interpolated location of the maximum, and his experiments showed that this provides a substantial improvement to matching and stability. His approach uses the Taylor expansion (up to the quadratic terms) of the scale-space function, $D(x, y, \sigma)$, shifted so that the origin is at the sample point.

$$
D(\mathrm{x})=D+\frac{\partial D^{T}}{\partial \mathrm{x}} \mathrm{x}+\frac{1}{2} \mathrm{x}^{\mathrm{T}} \frac{\partial^{2} D}{\partial \mathrm{x}^{2}} \mathrm{x}
$$

Where $D$ and its derivatives are evaluated at the sample point and $x=(x, y, \sigma) T$ is the offset, from this point. The location of the extremes, ' $x$, is determined by taking the derivative of this function with respect to $x$ and setting it to zero, giving

$$
\mathrm{s}=-\frac{\partial^{2} D}{\partial \mathrm{x}^{2}} \frac{\partial D}{\partial \mathrm{x}}
$$

As suggested by Brown, the Hessian and derivative of $D$ are approximated by using differences of neighboring sample points. The resulting $3 \times 3$ linear system can be solved with minimal cost. If the offset ${ }^{2} x$ is larger than 0.5 in any dimension, then it means that the extreme lies closer to a different sample point. In this case, the sample point is changed and the interpolation performed instead about that point. The final offset ${ }^{2} x$ is added to the location of its sample point to get the interpolated estimate for the location of the extremum. The function value at the extremum, $D\left({ }^{\wedge} x\right)$, is useful for rejecting unstable extrema with low contrast. For the experiments, all extrema with a value of $\left|\mathrm{D}\left({ }^{\wedge} \mathrm{x}\right)\right|$ less than 0.03 were discarded (as before, we assume image pixel values in the range $[0,1])$. The key point selection is shown in figure 4

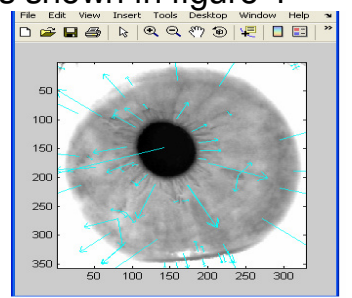

FIGURE 4: Key points detection

By assigning a consistent orientation to each keypoint based on local image properties, the keypoint descriptor can be represented relative to this orientation and therefore achieve invariance to image rotation.

\subsubsection{Key-Point Matching}

The best candidate match for each key point is found by identifying its nearest neighbor in the database of key points from training images. The nearest neighbor is defined as the keypoint with minimum Euclidean distance for the invariant descriptor. However, many features from an image will not have any correct match in the training database because they arise from background clutter or were not detected in the training images. Therefore, it would be useful to have a way to discard features that do not have any good match to the database. A global threshold on distance to the closest feature does not perform well, as some descriptors are much more discriminative than others. A more effective measure is obtained by comparing the distance of the closest neighbor to that of the second-closest neighbor. If there are multiple training images of the same object, then we define the second-closest neighbor as being the closest neighbor that is known to come from a different object than the first, such as by only using images known to contain different objects. This measure performs well because correct matches need to have the closest neighbor significantly closer than the closest incorrect match to achieve reliable matching. For false matches, there will likely be a number of other false matches within similar distances due to the high dimensionality of the feature space. We can think of the second-closest match as providing an estimate of the density of false matches within this portion of the feature space and at the same time identifying specific instances of feature ambiguity. 


\subsubsection{Clustering With Hough Transform}

Each of our keypoints specifies 4 parameters: 2D location, scale, and orientation, and each matched keypoint in the database has a record of the keypoint's parameters relative to the training image in which it was found. Therefore, we can create a Hough transform entry predicting the model location, orientation, and scale from the match hypothesis. This prediction has large error bounds, as the similarity transform implied by these 4 parameters is only an approximation to the full 6 degree of-freedom pose space for a 3D object and also does not account for any non rigid deformations. Therefore, we use broad bin sizes of 30 degrees for orientation, a factor of 2 for scale, and 0.25 times the maximum projected training image dimension (using the predicted scale) for location. To avoid the problem of boundary effects in bin assignment, each keypoint match votes for the 2 closest bins in each dimension, giving a total of 16 entries for each hypothesis and further broadening the pose range.

\section{RESULTS}

Figures below shows the results obtained by applying SIFT

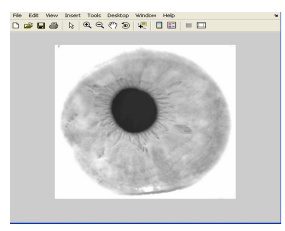

Fig. 5 : Original Image

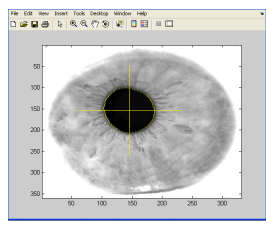

Fig. 6 centriod detection

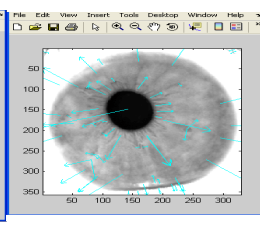

Fig. 7 : Key points detection

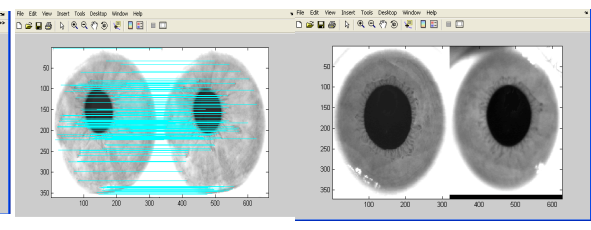

Fig.8 : Image matching Fig 9 : Match not found

\section{CONCLUSION}

Iris recognition system has been developed steadily with the help of MATLAB and some mathematical calculations, however limitations such as blur and dynamically taken images make it impossible to achieve perfect naturalness to combat this, we need to take images in ultraviolet environment. After getting image from the user the system will apply Hough transform detector technique to distinguish between pupillary and iris part of human eye, system applied various inbuilt MATLAB functions and mathematical calculations to encircle outer part of pupil that is inner part of iris and will mark the outer part of iris.

\section{REFERENCES}

[1] Y.Belganoui, J-C.Guézel and T.Mahé, « La biométrie, sésame absolu ...», Industries et Techniques, France, $\mathrm{n}^{\circ} 817$, July 2000.

[2] M.M.Gifford, D.J. McCartney and C.H.Seal, « Networked biometrics systems: requirements based on iris recognition », BT Technol. Journal, Vol. 17, April 1999.

[3] A.Bertillon, « la couleur de l'iris », Revue scientifique, France, 1885.

[4] J.Daugman, " High confidence personal identification by rapid video analysis of iris texture », Proc. Of the IEEE, International Carnahan conf. on security technology, 1992.

[5] R.P.Wildes, J.C. Asmuth, G.L. Green and S.C. Hsu, « A system for automated iris recognition », IEEE paper, 1994.

[6] W.W.Boles, " a security system based on human iris identification using wavelet transform ", First international conference on knowledge-based intelligent electronic systems, Adelaide, Australia. Ed, 21-23 may 1997. 
[7] R.Sanchez-Reillo, C.Sanchez-Avila and J-A.Martin- Pereda, "minimal template size for iris recognition », Proc. BMES/EMBS Conf., IEEE Publication, Atlanta, October 1999.

[8] J.Daugman, «High confidence personal identification by rapid video analysis of iris texture ", IEE Conf. Publication , European convention on security and detection, 16-18 May 1995.

[9] D.Gabor, «Theory of communication », Journal IEE,Vol.93, no III, 1946.

[10] J.Daugman, « Uncertainty relation for resolution in space, spatial frequency, and orientation optimized by two-dimensional visual cortex filters », Journal of optical society of America, Vol. 2, 1985.

[11] D.E.Benn, M.S.Nixon and J.N.Carter, « Robust eye extraction using H.T. », AVBPA'99.

[12] J.P.Havlicek, J.W.Havlicek and A.C.bovik, «the analytic image », IEEE Journal, 1997.

[13] D.Gabor, « Theory of communication », J. inst. Elect. Eng. London, Vol. 93, n III, 1946.

[14] A.C.Bovik, «The handbook of image processing », Ed. Bovik.

[15] J.P.Havlicek, D.S.Harding, and A.C.Bovik, «Discrete quasi eigenfunction approximation for AM-FM image analysis », Proc. Of the IEEE Int. Conf. on Image Processing, 1996.

[16] H. Davson, The Physiology of the Eye, 2nd ed. Boston, MA: Little, Brown \& Co., 1963.

[17] A. W. Drake, Fundamentals of Applied Probability Theory. New York: McGraw-Hill, 1986.

[18] R. O. Duda and P. E. Hart, Pattern Classification and Scene Analysis. New York: Wiley, 1973.

[19] R. A. Fisher, "The use of multiple measurements in taxonomic problems," Annals Eugenics, vol. 7, no. 2, pp. 179-188, 1936.

[20] L. Flom and A. Safir, "Iris recognition system," U.S. Patent 4641 349, 1987.

[21] D. Gabor, "Theory of communication," J. Inst. Elect. Eng., vol. 93, pp. 429-459, 1946.

[22] S. B. Grimes and I. Kershner, Never Say Never Again, Warner Brothers, 1983.

[23] K. Hanna, R. Mandelbaum, L. Wixson, D. Mishra, and V. Paragana, "A system for nonintrusive human iris acquisition," Machine Vision Applications, Tokyo, Japan, 1996, pp. 200-203.

[24] J. P. Holmes, L. J. Wright, and R. L. Maxwell, "A performance evaluation of biometric identification devices," SIL Albuquerque, NM, Tech. Rep. SAND91- 0276, 1991.

[25] B. K. P. Horn, Robot Vision. Cambridge, MA: MIT Press, 1986.

[26] P. Horowitz and W. Hill, The Art of Electronics, $2^{\text {nd }}$ ed. New York: CUP, 1988.

[27] P. V. C. Hough, "Method and means for recognizing complex patterns," U.S. Patent 3069 654, 1962.

[28] J. Illingworth and J. Kittler, "A survey of the Hough transform," Comput. Vision, Graph. Image Processing, vol. 44, pp. 87-116, 1988. 
[29] B. Jahne, Digital Image Processing, 2nd ed. Berlin: Springer- Verlag, 1993.

[30] N. S. Jayant and P. Noll, Digital Coding of Waveforms. Englewood Cliffs, NJ: PH-1984.

[31] F. A. Jenkins and H. E. White, Fundamentals of Optics. New York: McMillan, 1976.

[32] R. G. Johnson, "Can iris patterns be used to identify people," Los Alamos National Laboratory, CA, Chemical and Laser Sciences Division, Rep. LA-12331-PR, 1991.

[33] M. Kass, A Witkin, and D. Terzopoulos, "Snakes: Active contour models," in Proc. Int. Conf. Computer Vision, London, England, 1987, pp. 259-268.

[34] A. L. Kroeber, Anthropology. New York: Harcourt Brace Jovanovich, 1948.

[35] P. C. Kronfeld, "The gross anatomy and embryology of the eye," in The Eye, vol. 1, H. Davson, Ed. London: Academic, 1968, pp. 1-66.

[36] N. A. Macmillan and C. D. Creelman, Detection Theory: A User's Guide. Cambridge: Cambridge Univ. Press, 1991.

[37] A. Malickas, personal communication, 1994.

[38] I. Mann, The Development of the Human Eye. New York: Grune and Stratton, 1950.

[39] D. Marr, Vision. New York: Freeman, 1982.

[40] B. Miller, "Vital signs of identity," IEEE Spectrum, vol. 31, pp. 22-30, Feb. 1994.

[41] D. Miller, Ophthalmology. Boston, MA: Houghton Mifflin, 1979.

[42] F. W. Newell, Ophthalmology Principles and Practice, 7th ed. St. Louis, MO: Mosby, 1991.

[43] G. Olivier, Practical Anthropology. Springfield, IL: Charles C. Thomas, 1969.

[44] W. K. Pratt, Digital Image Processing. New York: Wiley, 1978.

[45] A. Samal and P. A. lyengar, "Automatic recognition and analysis of human faces and facial expressions: A survey," Pattern Recognit., vol. 25, pp. 65-77, 1992.

[46] J. E. Siedlarz, "Iris: More detailed than a fingerprint," IEEE Spectrum, vol. 31, p. 27, Feb. 1994.

[47] P. Sinha, "A head mounted display," Bachelor's thesis, Department of Electrical Engineering and Computer Science, Massachusetts Institute of Technology, Cambridge 